\title{
THE MAY MEETING IN FRESNO
}

The four hundred eighty-first meeting of the American Mathematical Society was held at Fresno State College, Fresno, California, on Saturday, May 3, 1952. Approximately 75 persons attended, including the following 59 members of the Society:

H. L. Alder, H. M. Bacon, W. G. Bade, A. K. Bell, L. D. Berkovitz, E. W. Beth, F. C. Biesele, W. W. Bledsoe, L. M. Blumenthal, F. H. Brownell, J. G. van der Corput, P. H. Daus, Douglas Derry, D. J. Ewy, F. D. Faulkner, G. E. Forsythe, N. S. Free, R. M. Hayes, M. R. Hestenes, P. G. Hodge, P. J. Kelly, L. D. Kovach, Cornelius Lanczos, B. J. Lockhart, Charles Loewner, M. W. Maxfield, L. F. Meyers, E. D. Miller, F. R. Morris, T. S. Motzkin, Ivan Niven, L. J. Paige, T. K. Pan, R. S. Phillips, D. H. Potts, W. T. Puckett, F. M. Pulliam, C. H. Rawlins, Jr., R. M. Redheffer, J. B. Robinson, R. M. Robinson, Leo Sario, M. M. Schiffer, I. J. Schoenberg, Abraham Seidenberg, R. G. Selfridge, M. A. Shader, Max Shiffman, W. H. Simons, T. H. Southard, M. L. Stein, Robert Steinberg, C. B. Tompkins, J. L. Ullman, W. R. Wasow, M. A. Weber, František Wolf, H. H. Wolfenden, F. H. Young.

By invitation of the Committee to Select Hour Speakers for Far Western Sectional Meetings, Professor I. J. Schoenberg, of the Institute for Numerical Analysis, National Bureau of Standards, Los Angeles, delivered an address entitled On smoothing operations and related topics. Professor Schoenberg was introduced by Professor Ivan Niven. There were sessions for contributed papers in the morning and afternoon, presided over by Professors F. R. Morris, H. M. Bacon, and P. H. Daus. Following the meeting there was a guided tour of Pine Flat Dam, now under construction.

Following are abstracts of papers presented at the meeting. Papers with abstract numbers followed by " $t$ " were presented by title. Paper number 490 was presented by Professor Steinberg, number 498 by Professor Kelly, and number 502 by Dr. Forsythe. Dr. Reid was introduced by Dr. J. W. Odle and Mr. Bennett by Professor F. C. Biesele.

\section{Algebra AND Theory of Numbers}

\section{3t. D. R. Morrison: Boolean product rings.}

A Boolean product $R \circ B$ is defined for every ring $R$ and every Boolean ring $B$, differing from the tensor product in that the left distributive law, $r \circ\left(b_{1}+b_{2}\right)=r \circ b_{1}$ $+r_{2} b$, holds only if $b_{1} b_{2}=0$ or $2 r=0$. Every nonzero element of $R \circ B$ is uniquely expressible in the form $\sum_{i=1}^{n} r_{i} \circ b_{i}$, where $r_{j} \neq r_{i} \neq 0, b_{i} \neq 0, b_{i} b_{j}=0$ for $j \neq i$. Where they are meaningful, the commutative and associative laws and the distributive laws with respect to weak direct sums apply to the Boolean product operation, and $G F(2)$ is a unit with respect to the operation. The idempotent Boolean ring $(R \circ B)^{0}$ 
(see Bull. Amer. Math. Soc. Abstract 58-1-52) is isomorphic to $R^{0} \circ B$. If $R$ is biregular, so is $R \circ B$ and relations are determined between the ideals of $R, B$, and $R \circ B$. For any Boolean ring $B$, and any prime, $p, G F(p) \circ B$ is a $p$-ring. Conversely, for any $p$-ring $R, R^{0}$ is a Boolean ring and $R$ is isomorphic to $G F(p) \circ R^{0}$. This establishes a correspondence between Boolean rings and p-rings, which is biunique up to an isomorphism. (Received March 13, 1952.)

\section{T. S. Motzkin: The multi-index transportation problem.}

Let $x_{\alpha_{1} \alpha_{2}} \cdots \alpha_{n}, \alpha_{i}=1, \cdots, a_{i}\left(a_{i}>1, i=1, \cdots, n\right)$, be unknowns. For a given $r, r=1, \cdots, n-1$, make all choices $k=\left(1 \leqq i_{1}<\cdots<i_{n-r} \leqq n ; \alpha_{i_{1}}, \cdots, \alpha_{i_{n-r}}\right)$. It is shown that the extreme solutions of the system $\sum x_{\alpha_{1} \alpha_{2} \cdots \alpha_{n}}=c_{k}, x_{\alpha_{1} \alpha_{2} \cdots \alpha_{n}} \geqq 0$ (the summation running over all $\alpha_{i}$ with $i \neq i_{1}, \cdots, i_{n-r}$ ) lie, for every given system of constants $c_{k}$, in the ring generated by the $c_{k}$, if and only if $r=1$ and no more than two $a_{i}$ exceed 2. (Received March 13, 1952.)

\section{5t. R. M. Redheffer: Power series and algebraic numbers.}

Suppose $f(z)=\sum a_{n} z^{n}$ is rational, is regular at $z=0$, has algebraic coefficients, and is not a polynomial. Let $m$ be the multiplicity of that root in the denominator of $f$ which has maximum multiplicity. Then (apart from a set of at most $m$ values of $z$ ) the value of the function $g(z)=\sum a_{n} z^{n} / n$ ! is transcendental when the value of $z$ is algebraic. (Received March 17, 1952.)

476. Abraham Seidenberg: Some basic theorems in differential algebra (characteristic p, arbitrary).

The theorem of the primitive element, the chain theorem, and the Hilbert Nullstellensatz, all appropriately formulated, are established for differential algebra of arbitrary characteristic. Simplified proofs of the known results in the case of characteristic 0 are included. The theory of transcendency and the theory of separability are also considered. (Received March 13, 1952.)

\section{ANALysis}

477t. R. E. Bellman: Some mock hydrodynamical equations. Preliminary report.

The equation (1) $u_{t}+u u_{x}=\mu u_{x x}$ was used by J. M. Burgers, Proc. Acad. Sci. Amsterdam vol. 43 (1940), as a mathematical model to illustrate the theory of turbulence. It was shown by E. Hopf, Comm. Pure and Applied Math. vol. 3 (1950), that this equation could be linearized by means of the change of variable, $u=-2 \mu(\log \phi)_{x}, \phi$ satisfying the equation $\mu \phi_{x x}=\phi_{t}$. It is shown in the present paper that $u=-2 \mu(\log \phi)_{x}, v=-2 \mu(\log \phi)_{y}$ furnishes a solution to the two-dimensional analogue of (1), $u_{t}+u u_{x}+v u_{y}=\mu\left(u_{x x}+u_{y y}\right), v_{t}+u v_{x}+v v_{y}=\mu\left(v_{x x}+v_{y y}\right)$, if $\phi$ satisfies the equation $\mu\left(\phi_{x x}+\phi_{y y}\right)=\phi_{t}$, and similarly for three dimensions. These explicit solutions may now be used, following the one-dimensional treatment of Hopf, to determine rigorously the behavior of the solutions as $\mu \rightarrow 0$. If the pressure terms are included in the hydrodynamical equations, the equation for $\phi$ is $\mu\left(\phi_{x x}+\phi_{y y}\right)+p \phi=\phi_{t}$. (Received March 17, 1952.)

478. F. H. Brownell: Vector analysis on real separable Hilbert space. Preliminary report. 
Consider complex-valued functions $\psi(f)$ over $f \in l_{2}$, the square summable real sequences and the prototype of real separable Hilbert space. Following Hille, for suitable functions $\psi$ one can define the notion of a vector derivative $\nabla \psi(f)$, where for $\psi$ real $\nabla \psi(f) \in l_{2}$ with $\|u\|^{-1}|\psi(f+u)-\psi(f)-(\nabla \psi(f), u)| \rightarrow 0$ as $\|u\| \rightarrow 0, u \in l_{2}$. This can be interpreted in terms of the $l_{2}$ coordinate derivatives, and using basis coordinates the generalized Laplacian $\nabla^{2}$ can be defined invariant under change of basis. In order to integrate on $l_{2}$ and still have an abelian group, it seems $l_{2}$ must be reduced to some square summable infinite torus $X, X=\left\{x \in l_{2} \mid-h_{n}<x_{n} \leqq h_{n}\right.$ each $\left.n\right\}$ with $\sum^{\infty}\left(h_{n}\right)^{2}$ $<+\infty$ required, a modification of Jessen's torus integration. This gives a locally compact abelian topological group, which thus possesses Fourier transforms with inverses, and one can verify the expected relationship of Fourier transforms to partial derivatives with respect to $l_{2}$ coordinates, as well as to the generalized Laplacian $\nabla^{2}$. (Received March 13, 1952.)

479t. R. S. Phillips: On the generation of semi-groups of linear operators.

Let $T(t)$ be a semi-group of linear bounded transformations on a Banach space $\mathfrak{X}$ to itself, strongly continuous on $[0, \infty)$ with $T(0)=I$. Set $\omega(\xi)=\log \|T(\xi)\|$ and define $S(\omega)$ to be the Banach algebra of set functions $\alpha$ where $\|\alpha\|=\int_{0}^{\infty} \exp [\omega(\xi)]|d \alpha|$ and the product is given by convolution. Let $\omega_{0}=\inf \omega(\xi) / \xi$ and set $\phi(\lambda, \alpha)$ $=\int_{0}^{\infty} \exp (\lambda \xi) d \alpha$ for $\operatorname{Re}(\lambda) \leqq \omega_{0}$. Assume further that $\lim _{\delta \rightarrow 0} \sup _{\xi \geqq 0}[\omega(\xi)-\omega(\xi+\delta)]$ $<\infty$. Then a necessary and sufficient condition that $\alpha(t, \cdot)$ be a semi-group of monotone nondecreasing functions in $\subseteq(\omega)$ (with $\phi[\lambda, \alpha(t, \cdot)] \rightarrow 1$ as $t \rightarrow 0$ uniformly in every bounded subset of $\left.\operatorname{Re}(\lambda) \leqq \omega_{0}\right)$ is that $t^{-1} \log \phi[\lambda, \alpha(t, \cdot)]=m \lambda+\int_{0}^{\infty}\left(\exp \left[\left(\lambda-\omega_{0}\right) \xi\right]\right.$ $-1) d \psi+a$ where $m \geqq 0, a$ is real, and $\psi(\xi)$ is monotone nondecreasing on $(0, \infty)$ satisfying the conditions $\int_{0}^{1} \xi d \psi<\infty$ and $\int_{1}^{\infty} \exp \left[\omega(\xi)-\omega_{0} \xi\right] d \psi<\infty$. For a semi-group $\alpha(t, \cdot)$ of this description $S(t)=\int_{0}^{\infty} T(\xi) d \alpha(t, \xi)$ is again a semi-group of linear bounded transformations on $\mathfrak{X}$ to itself, strongly convergent to $I$ at $t=0$. If $A$ is the infinitesimal generator of $T(t)$ and $B$ that of $S(t)$, then for $x$ in the domain of $A, B x=m A x$ $+\int_{0}^{\infty}\left[\exp \left(-\omega_{0} \xi\right) T(\xi) x-x\right] d \psi+a x$. In addition if $\lambda_{0}$ belongs to the spectrum of $A$, then $t^{-1} \phi\left[\lambda_{0}, \alpha(t, \cdot)\right]$ belongs to the spectrum of $B$. (Received December 18, 1951.)

\section{R. S. Phillips: Perturbation theory for semi-groups of linear} operators.

Those properties of a semi-group of linear bounded operators strongly continuous to the identity at the origin which persist under a linear bounded perturbation of the infinitesimal generator are called stable. It is shown that the property of being a semi-group or a group is itself stable. If $A$ generates $T(s)$ and $A+B$ (with $B$ bounded) generates $S(s)$, then $S(s)=\sum_{n=0}^{\infty} S_{n}(s)$ where $S_{0}(s)=T(s)$ and $S_{n}(s)$ $=\int_{0}^{a} T(s-\sigma) B S_{n-1}(\sigma) d \sigma$. If $T(s)$ is uniformly continuous for $s>0$, then so is $S(s)$. However the property of being eventually uniformly continuous is not stable. If $T(z)$ is holomorphic in a sector and bounded near the origin in every sub-sector, then the same is true of $S(z)$. If the resolvent of $A$ is completely continuous, then so is the resolvent of $A+B$. It is possible to develop a "finite" elementary divisor theory for $A$ when the resolvent of $A$ is completely continuous. If $A(s)=A+B(s)$ where $A$ is the infinitesimal generator of a semi-group of operators and $B(s)$ is a strongly continuously differentiable family of bounded linear operators, then there exists a unique strongly-continuous one-parameter family of bounded linear operators $U(s)$ such that $d U(s) x / d s=A(s) U(s) x$ for all $x \in D(A)$ and such that $U(0)=I$. (Received February $22,1952$. 


\section{1t. R. M. Redheffer: $A$ basis theorem for partial differential equa- tions.}

Let $X=(x, y, z, \cdots, w)$ denote the independent variables, $U=U(X)$ the dependent variable, and $V=\left(U, U_{x}, \cdots\right)$ a finite-dimensional vector with $U$ and some of its partial derivatives as components. A partial differential equation is an equation of form $f(X, V)=0$. If $f$ is continuous and if a linearly closed family of solutions $U_{\alpha}$ solves $f(X, V)=0$, then there is a finite system of linear homogeneous partial differential equations $A \cdot V=0, B \cdot V=0, \cdots$, such that (1) each $U_{\alpha}$ solves the system and (2) each solution of the system solves $f(X, V)=0$. The result follows from simple properties of finite-dimensional vector spaces. (Received March 17, 1952.)

482t. R. M. Redheffer: Differential equations and the Huygens principle.

Let $U(x, y)=\left(1 /(2 \pi)^{1 / 2}\right) \int_{-\infty}^{\infty} e^{i t y}[\Lambda(t)]^{x} F(t) d t$, where $\Lambda$ is measurable, $\Lambda \neq 0, \Lambda \neq \infty$; and let $U$ satisfy one and the same partial differential equation for all $F$ giving sufficiently strong convergence of the integral. Then $\log \Lambda(t)$ is equal almost everywhere to an algebraic function of $t$, and the functions $U$ all are solutions of one and the same linear homogeneous partial differential equation with constant coefficients. (Received March 17, 1952.)

\section{R. M. Redheffer: Operators and the Huygens principle.}

Let $\phi_{a b}$ be a class of operators which transfer a disturbance $f(x, y)$ from $x=a$ to $x=b: f(b, y)=\phi_{a b} f(a, y)$. These give an abstract formulation of Huygens' principle in one dimension. Assuming linearity, a closure condition $\phi_{a c}=\phi_{a b} \phi_{b c}$, and boundedness in two senses, representation theorems are given. The simplest result (for "uniform media") is $\phi_{a b}=T^{-1} \Lambda^{b-a} T$, where $T$ is the Fourier transform in the $L^{2}$ sense, $T^{-1}$ the inverse transform in the $L$ sense, and $\Lambda(u) \in L^{p}$ for all $p$. (Received March 17,1952.)

\section{W. P. Reid: On the relaxation method of solving differential equations.}

Allen and Severn (Quarterly Journal of Mechanics and Applied Mathematics vol. 4, Part 2 (1951) p. 209) have stated that "It is, in fact, only to problems of jury type that the relaxation method is directly applicable," where jury type means equal number of boundary conditions at each of two points. This statement is incorrect. The relaxation method may be applied directly to solve differential equations of any order with the $n$ boundary conditions distributed in any way among $P$ points $(1 \leqq P \leqq n)$. Their method of raising the order of the differential equations to make them of jury type is a method of fooling oneself and results primarily in extra work. These remarks, by the way, apply also to problems governed by partial differential equations. Also, the applications of the relaxation method have apparently been restricted so far to bounded regions. This is not necessary. The region may be unbounded, and a condition may or may not be specified at infinity. (Received March 10, 1952.) I.

485t. Leo Sario: An extremal method on arbitrary Riemann surfaces.

A method is indicated for constructing a function which minimizes a prescribed expression $m$ in a given class $F$ of functions defined on an arbitrary Riemann surface 
$R$. Consider the class $\{P\}$ of analytic functions with the development $P=p+i \bar{p}$ $=1 / z+\sum_{\nu=1}^{\nu=\infty} a_{\nu} z^{\nu}$ in a fixed parameter disc $K:|z| \leqq 1$, and with a single-valued real part on $R$. Write $\alpha=\operatorname{Re}\left(a_{1}\right)$ and denote by $\beta$ the (ideal) boundary of $R$. Let $\lambda$ be a real parameter, $-1 \leqq \lambda \leqq 1$. Then (1) there is in $\{P\}$ a unique principal function $P_{\lambda}=p_{\lambda}+i \overline{p_{\lambda}}=1 / z+\sum_{\nu=1}^{\nu=\infty} a_{\lambda \nu} z^{\nu}$ which minimizes the expression $m_{\lambda}=\lambda \cdot 2 \pi \alpha+\int_{\beta} p d \bar{p}$. (2) This minimum is $m_{\lambda}\left(p_{\lambda}\right)=(\pi / 2)\left[(1+\lambda)^{2} \alpha_{1}-(1-\lambda)^{2} \alpha_{-1}\right]$ where $\alpha_{\lambda}=\operatorname{Re}\left(a_{\lambda 1}\right)$. (3) $m_{\lambda}(p)-m_{\lambda}\left(p_{\lambda}\right)=D\left(p-p_{\lambda}\right)$, the Dirichlet integral. (4) $P_{\lambda}=(1+\lambda) P_{1} / 2+(1-\lambda) P_{-1} / 2$. (5) $\alpha_{1} \leqq \alpha \leqq \alpha_{-1}$ for functions $p$ with $\int_{\beta} p d \bar{p} \leqq 0$. Definition: the $P$-span $\sigma_{P}=\alpha_{-1}-\alpha_{1}$. (6) $\sigma_{P}=\min (2 / \pi) \int_{\beta} p d \bar{p}=(2 / \pi) \int_{\beta} p_{0} d \bar{p}_{0}$. (7) $1 / \sigma_{P}=\min (1 / 2 \pi) D(f)=(1 / 2 \pi) D\left(f_{0}\right)$ where $(f)$ is the class of single-valued harmonic functions $f$ on $R$ with the development $f+i \bar{f}=\sum b_{\nu} z^{\nu}$ in $K, \operatorname{Re}\left(b_{1}\right)=1$, and $f_{0}+\overline{i f_{0}}=\left(P_{-1}-P_{1}\right) / \sigma_{P}$. (8) The class $\{P\}$ degenerates to a single function if and only if the $P$-span vanishes. These results remain valid for the subclass $\{Q\}$ of $\{P\}$, defined by the restriction $\int d \bar{p}=0$ along each dividing cycle on $R$, if the $P$-span is replaced by the corresponding $Q$-span. For planar surfaces, $\{Q\}$ consists of single-valued analytic functions and the $Q$-span coincides with Schiffer's span. Earlier results concerning the span appear as special cases. (Received March 10, 1952.)

\section{6t. Leo Sario: An extremal method on arbitrary Riemann surfaces. II.}

The boundedness of $p_{-1}-p_{1}$ and $q_{-1}-q_{1}\left(Q_{-1}-Q_{1}\right.$ for planar surfaces) is used to establish a unified approach to classification problems on Riemann surfaces. The following abbreviations are employed: $H$, harmonic single-valued nonconstant; $K$, harmonic single-valued nonconstant, such that the conjugate function has no periods along dividing cycles; $A$, analytic single-valued nonconstant; $W$, meromorphic nonconstant; $B$, bounded; $D$, with a finite Dirichlet integral; $E$, omitting a set of values with positive area. By combinations of letters, classes of functions are denoted with the corresponding combinations of properties. Let $O_{F}$ be the class of Riemann surfaces not admitting functions belonging to a given class $F$. Denote by $S_{P}$ and $S_{Q}$ the classes of surfaces with vanishing $P$-span or $Q$-span, respectively. Then (1) $O_{H B} \subset O_{H B D}=O_{H D}=S_{P}$, (2) $O_{K B} \subset O_{K B D}=O_{K D}=S_{Q}$, (3) $O_{W E}=O_{A B} \subset O_{A D}$. Earlier results, concerning $H$ and $A$, are included in these relations. The last equality (1) states that there are functions $H D$ on $R$ if and only if the $P$-span vanishes. The relations (2), applied to planar surface, express existence properties of single-valued analytic functions. (Received March 10, 1952.)

\section{7t. Leo Sario: Construction of functions with prescribed properties on Riemann surfaces.}

Let $G$ be the complement $R-D$ of a compact subdomain $D$ of an open Riemann surface $R, D$ being bounded by a finite set $\alpha$ of analytic Jordan curves. The (ideal) boundary of $R$ is denoted by $\beta$. Let $v$ be a single-valued function, harmonic on $\alpha$, let $\lambda$ be a real parameter, $-1 \leqq \lambda \leqq 1$. Denote by $\{u\}$ the class of single-valued harmonic functions in $G$ with $u=v$ on $\alpha, \int_{\alpha} d \bar{u}=0$. There exists in $\{u\}$ a unique function $u_{\lambda}$ which minimizes the expression $\int_{\beta} u d u+\lambda \int_{\alpha} u d u$. The function $u_{\lambda}$ is associated with $v$ by a normal linear operator $L_{\lambda}$. This theorem remains valid for the subclass $\left\{u^{0}\right\}$ of $\{u\}$ defined by the restriction $\int d u=0$ along every dividing cycle. Both $u_{\lambda}$ and $u_{\lambda}^{0}$ are constructed by the extremal method reported in the above abstracts. The linear operator method (Trans. Amer. Math. Soc. March 1952) leads to the following theorem. Let $s$ be a single-valued real function on $D$, harmonic on $\alpha$. The condition 
$\int_{\alpha} d \bar{s}=0$ is necessary and sufficient for the existence of a function $p$ on $R$ such that (1) $p-s$ is harmonic on $D$, (2) $\int_{\beta} u d u+\lambda \int_{\alpha} u d u$ is minimized by $p$ among all harmonic functions $u$ in $G$ with $u=p$ on $\alpha$ and $\int d \bar{u}=0$ along $\alpha$ (or else along every dividing cycle). (Received March 10, 1952.)

\section{Leo Sario: Capacity of the boundary and of a boundary com-} ponent. I.

Let $h(z)$ be a harmonic function in $|z| \leqq 1$ with $h(0)=0$. Write $s=\log |z|+h(z)$. Denote the curves $|z|=e^{-1},|z|=1$ by $a$ and $b$, respectively. Lemma: $\min _{b} s-\min _{a} s$ $\leqq 1$. On an arbitrary open Riemann surface $R$ with (ideal) boundary $\beta$, let $\{S\}$ be the class of analytic functions with the development $S=s+i \bar{s}=\log z+\sum_{y=1}^{y=\infty} a_{y} z^{\nu}$ in a parameter disc $|z| \leqq 1$, and with a single-valued real part $s$. There is in $\{S\}$ a unique principal function $S_{\beta}$ such that $\min \int_{\beta} s \bar{s}=\int_{\beta} s_{\beta} d_{\bar{s} \beta}$. Definition: the capacity of $\beta$ is $C_{\beta}=e^{-k_{\beta}}$ where $k_{\beta}=(1 / 2 \pi) \int_{\beta} s_{\beta} d_{\bar{s} \beta}$. For planar domains, $C_{\beta}$ coincides with the conventional (logarithmic) capacity. Consider the class $\{U\}$ of analytic functions with a single-valued modulus on $R$ and with the development $U=\sum_{\nu=1}^{\nu, \infty} b_{\nu} z^{\nu}\left(b_{1}=1\right)$ in $|z| \leqq 1$. In $\{U\}$, there is a unique principal function $U_{\beta}=e^{S_{\beta}}$ such that $\min D(U)$ $=D\left(U_{\beta}\right)=\pi C_{\beta}^{-2}$. Suppose now $\beta$ is analytic. For every analytic function $w$ with single-valued modulus on $R$ such that $|w| \leqq C_{\beta}^{-1}$, the relations $|w|<\left|U_{\beta}\right|, w^{\prime}(0)<1$ hold unless $w \equiv e^{i \alpha} U_{\beta}$. Denote by $M$ the class of nonconstant analytic functions with single-valued modulus on an arbitrary Riemann surface $R$. The subclasses of functions with bounded modulus or with finite Dirichlet integral are denoted by $M B$ and $M D$, respectively. There are functions $M B$ or $M D$ on $R$ if and only if $C_{\beta}>0$. (Received March 10, 1952.)

489t. Leo Sario: Capacity of the boundary and of a boundary component. II.

Let $\gamma$ be a fixed boundary component of an arbitrary Riemann surface $R$ with boundary $\beta$. Consider the subclass $\{T\}$ of $\{S\}$ defined by $\int d \bar{s}=2 \pi$ along every cycle separating $\gamma$ from $z=0$, and $\int d \vec{s}=0$ along every other cycle. There exists in $\{T\}$ a unique principal function $T_{\gamma}$ such that $\min \int_{\beta} t d \bar{t}=\int_{\beta} t_{\gamma} d \bar{t}_{\gamma}$. Definition: the capacity of $a$ boundary component $\gamma$ is $C_{\gamma}=e^{-k \gamma}$ with $k_{\gamma}=(1 / 2 \pi) \int_{\beta} t_{\gamma} d \bar{t}_{\gamma}$. Let $\{V\}$ be the subclass of $\{U\}$ defined by $\int d$ arg $u=2 \pi$ along cycles separating $\gamma$ from $z=0, \int d$ arg $u=0$ along other cycles. On a planar $R$, there is in $\{V\}$ a unique principal function $V_{\gamma}$ $=e^{T \gamma}$ such that $\min D(V)=D\left(V_{\gamma}\right)=\pi C_{\gamma}^{-2}$. There exist on $R$ univalent bounded or univalent Dirichlet bounded nonconstant functions if and only if the capacity of at least one boundary component is positive. (Received March 10,1952.)

490. Robert Steinberg and R. M. Redheffer: Simultaneous trigonometric approximation.

Given real functions $f(x)$ and $g(x)$ in $L^{2}$, how small can $I=\int_{0}^{b} \mid f(x)+i g(x)$ $-\sum_{k=0}^{n} a_{k}^{(n)} e^{i n x} \mid 2 d x$ be made by a suitable choice of the real $a_{k}^{(n)}$ s, possibly subject to the constraint $\sum_{k=0}^{n} a_{k}^{(n) 2} \leqq M$ ? Expressions for the minimizing $a_{k}^{(n)}$ 's and the minimum $I$ are obtained. In the unconstrained case, $I$ can be made arbitrarily small as $n \rightarrow \infty$ only for conjugate functions $f(x)$ and $g(x)$ if $b=\pi$, but for arbitrary functions if $0<b<\pi$. The latter case leads to matric relations analogous to the ordinary Parseval identity. The following limitations on the approximation are obtained: (1) In general, there is no series approximation $\left(a_{k}^{(n)}\right.$ independent of $\left.n\right)$ for which $I \rightarrow 0$. If there is a series approximation, it is unique. (2) The following classes of function pairs $f, g$ 
are equivalent: (a) Those which admit a polynomial approximation with $I \rightarrow 0$ and $\sum_{k=0}^{n} a_{k}^{(n) 2} \leqq M$. (b) Those which admit a series approximation with $I \rightarrow 0$ and $\sum_{k=0}^{\infty} a_{k}^{2}$ $\leqq M$. Hence, in general, the approximation in (a) is not possible. (Received March 17, 1952.)

491. J. L. Ullman: Hankel determinants in function theory. Preliminary report.

Consider the power series $a_{0} z^{-1}+a_{1} z^{-2}+\cdots=f(z)$, and let $A(n, k)$ be the Hankel determinant of order $k$ with $a_{n+i+j-2}$ as the element in the $i$ th row and $j$ th column. To each sequence of integers $\left[k_{n}\right]$, associate the number $\left[k_{n}\right]^{\prime}=\lim \sup _{n \rightarrow \infty}\left|A\left(n, k_{n}\right)\right| \rho(n)$, $\rho(n)=1 /\left(k_{n}\left(n+k_{n}-1\right)\right)$. Hadamard proposed the problem of finding constructive means of determining the singularities of an analytic function from the coefficients of a single Taylor series expansion. In this direction he showed (J. Math. Pures Appl. (4) vol. 8, p. 101) that when the elements of $\left[k_{n}\right]$ are equal, $\left[k_{n}\right]^{\prime}$ is related to certain poles of $f(z)$. Polya (Math. Ann. vol. 99, p. 687) considers the case in which $\lim _{n \rightarrow \infty} k_{n} /\left(n+k_{n}\right)=\epsilon>0$, and shows that the $\left[k_{n}\right]^{\prime}$ are related to the entire set of singularities of $f(z)$. The large gap in these results suggested that $\left[k_{n}\right]^{\prime}$ for the case $\lim _{n \rightarrow \infty} k_{n}=\infty, \lim _{n \rightarrow \infty} k_{n} /\left(n+k_{n}\right)=0$, in which the rate of growth of $k_{n}$ is intermediate to the other cases, would be related to intermediate properties of $f(z)$. A precise relationship is established for functions $f(z)=\int_{0}^{1} d \mu(t) /(t-z), \mu(t)$ real and nondecreasing on $(0,1)$. Of independent interest is a generalization of the well known fact that if $A(0, k)$ is ultimately zero, then $\mu(t)$ is a step function; namely, it is shown that if $\lim _{k \rightarrow \infty}|A(0, k)|^{1 / k^{2}}=0$, then $\mu(t)$ has zero derivative on $(0,1)$, except for a set of measure zero. (Received April 15, 1952.)

492. W. R. Wasow: Asymptotic solution of the differential equation of hydrodynamic stability in a domain containing a transition point.

The differential equation $u^{(4)}+\sum_{j=1}^{4} a_{j}(x) u^{(4-j)}+\lambda^{2} \sum_{k=1}^{2} b_{k}(x) u^{(2-k)}=0$, with $b_{0}(0)=0, b_{0}^{\prime}(0) \neq 0, b_{2}(0) \neq 0$, is studied, in a complex neighborhood $S$ of $x=0$, for large values of the complex parameter $\lambda$. The $a_{j}(x)$ and $b_{k}(x)$ are supposed to be analytic in $S$. This problem is important in the study of the onset of turbulence in viscous flows. In a paper in Ann. of Math. vol. 49 (1948) pp. 852-871, the author constructed a fundamental system with known asymptotic behaviour in a ring-shaped domain surrounding the origin. It is now shown-independently of that paper-that the differential equation above possesses a fundamental system which, for large $\lambda$, is approximated, everywhere in $S$, by functions that can be simply and explicitly expressed in terms of solutions of the differential equation $y^{(4)}+\lambda^{2}\left(x y^{\prime \prime}+y\right)=0$. The asymptotic properties of this latter differential equation are known from another paper by the author (Ann. of Math. vol. 52 (1950) pp. 351-361). Thus it is possible to give a complete asymptotic analysis in $S$ of the more general differential equation, at least as far as the asymptotically leading terms are concerned. (Received March 3, 1952.)

\section{Applied Mathematics}

493. P. G. Hodge: Upper and lower bounds on the yield load of a square slab with a centered circular cutout. Preliminary report.

If an elastic-plastic body is loaded with a set of loads $P$, the collapse load corresponding to $P$ is defined as the smallest loads $\mu P$, where $\mu$ is a scalar, under which the body will exhibit unrestricted plastic flow. The present paper is concerned with a 
thin square slab with a centered circular cutout, subjected to uniform tensile stresses on each pair of opposite sides. The cutout factor $\lambda$ is defined as the largest multiplier $\lambda$ such that if $\left(T_{x}, T_{y}\right)$ represents any collapse load for the square without cutout, the square with a cutout will not collapse under any load less than $\left(\lambda T_{x}, \lambda T_{y}\right)$. Upper and lower bounds are then found for the cutout factor. The analysis is based on the assumptions of plane stress and Tresca's yield condition. (The results presented in this paper were obtained in the course of research conducted under Contract N7onr-35810 between the Office of Naval Research and Brown University.) (Received March 10, 1952.) tion.

494. Cornelius Lanczos: The smallest root of the characteristic equa-

In vibration problems the absolutely smallest eigenvalues of the generally nonsymmetric and nonreal matrix $A$ are of specific interest. Since the usual iterative procedures suppress the small eigenvalues in favor of the large ones, a preliminary inversion of $A$ is customarily demanded. The present procedure analyzes the problem in the orthogonal reference system of the Hermitian matrix $C=\widetilde{A}^{*} A$. The basic equation is written in the form $C x-\lambda \tilde{A}^{*} x=0$. The smallest eigenvector $x_{0}$ of $C$ is considered as the zeroth approximation of $x$. In first approximation $x=x_{0}+\lambda x_{1}$ where $x_{1}$ is the solution of a linear set of equations. The associated quadratic equation gives a (generally complex) $\lambda$ which is close to the absolutely smallest eigenvalue of $A$ and which can be further refined. This method, if applied to ordinary algebraic equations, yields a simple and convenient algorithm for approximating the absolutely smallest root of an algebraic equation. (Received March 4, 1952.)

\section{5t. R. M. Redheffer: Antennas with obstructions.}

If $T, T^{-1}$ are direct and inverse Fourier transform operators and $\Lambda(u)$ $=\exp i\left(k^{2}-u^{2}\right)^{1 / 2}, k=2 \pi / \lambda$, then $\Lambda^{-a} T t T^{-1} \Lambda^{a}$ operating on the original far pattern $F(u)$ gives the perturbed far pattern $F_{0}(u)$ due to an obstruction at $x=a$ characterized by the transmission function $t(y)$. Let an arbitrary obstacle be characterized by $T(u, v)$, the transmitted plane wave in "direction" $v=(2 \pi \sin \theta) / \lambda$ given a unit incident plane wave in "direction" $u$. Then $(2 \pi)^{1 / 2} F_{0}(v)=\Lambda^{-a} \int \Lambda^{a} T(u, v) F(u) d u$, and hence $t(y)$ exists if and only if $T(u, v)=\phi(u-v)$, for some $\phi$. A dense set in $t, f$, or $F$ yields a dense set in $F_{0}$, whence are obtained conditions of pattern realizability and methods of computation. The perturbed pattern is approximable as a finite sum of expressions $T \Lambda^{a} /(u-b)$, the transform being taken as a Sommerfeld contour integral. Another approach gives modified Bessel functions. For estimates of $F_{0}$ we have $F_{0}=F+\Lambda^{-a} H$, where $H \exp (-i m u)$, given explicitly, is slowly varying for some $m$. (Received March 17, 1952.)

\section{6t. R. M. Redheffer: Inhomogeneous dielectric media.}

Let $R$ be the complex reflection of a slab in which the dielectric constant is a continuous function of one coordinate, $\epsilon=\epsilon(x)$. If $z=-i(1+R) /(1-R)$ and $-k \epsilon z=u^{\prime} / u$, then $u$ satisfies $\left(u^{\prime} / \epsilon\right)^{\prime}+(2 \pi / \lambda)^{2} u=0$. Formulas are given for $R(z)$ in terms of $J(z)$, and for $z(x)$ for every termination from $z(x)$ for one. The polar form $z=r e^{i \theta}$ satisfies $r d \theta / d r=\tan \theta\left(\epsilon r^{2}-1\right) /\left(\epsilon r^{2}+1\right)$ when $\epsilon$ is real, whence is obtained a condition for realizability of a prescribed $z$. Regarding the medium as limit of thin uniform slabs gives a physical interpretation to the method of "product integration." (Received March 17, 1952.) 


\section{GEOMETRY}

\section{L. M. Blumenthal: Boolean geometry. I.}

A Boolean metric space $\mathcal{B}$ is obtained by associating with each pair of elements $p, q$ of a set an element $d(p, q)$ of a Boolean algebra as distance, so that $d(p, q)=0$ if and only if $p=q, d(p, q)=d(q, p)$, and $d(p, q)+d(q, r) \supset d(p, r) \quad(+, \supset$ denote Boolean addition and inclusion, in the wide sense, respectively). Boolean geometry studies the invariants of $\mathcal{B}$ under the group of congruences (i.e., distance-preserving mappings). Any Boolean algebra is a space $\mathbb{B}$ with $d(p, q)=p q^{\prime}+p^{\prime} q$ (juxtaposition and ' denoting Boolean product and complementation, respectively) and this paper begins the systematic study of these Boolean geometries by developing linearity notions and the theory of metric segments. Defining these in a suitable manner, they are shown to possess many properties of segments in ordinary metric spaces. Thus, (1) the length of a segment $S_{a}^{b}$ is the distance $d(a, b)$ of its end elements, (2) if $S_{a}^{b}$ $S_{b}^{c}$ are segments and $b$ is metrically between $a, c$ then their set-union is a segment $S_{a}^{0}$, and (3) for $m>4$, any $m$-tuple of $\mathcal{B}$ is on some segment if and only if each triple of the $m$-tuple has that property. (Received February 26, 1952.)

\section{P. J. Kelly and L. J. Paige: Symmetric perpendicularity in hilbert geometry.}

Define a family $F$ of curves as follows: Every curve $C$ in $F$ is a simple, closed, convex curve. If supporting lines at $p$ and $q$ on $C$ meet at $w$, and $\xi$ is any secant through $w$, cutting $C$ at $m$ and $n$, then supporting lines at $m$ and $n$ exist, intersecting on $p \times q$. The authors prove that the family $F$ consists of all ellipses and all nondegenerate triangles. This result is used to show that the hilbert geometries in which perpendicularity is symmetric are hyperbolic geometries. (Received February 18, 1952.)

499. T. K. Pan: Characterization of the Gaussian curvature of a surface.

Let $V_{n}$ be a hypersurface immersed in a Euclidean space $S_{n+1}$. Let $v$ be a vectorfield in $V_{n}$. Define the mean curvature of $v$ at a point $P$ as the square root of the sum of the squares of its $n-1$ principal curvatures at $P$ (the author's paper on Normal curvature of a vector-field, to appear soon in Amer. J. Math.). It is found that the hyperspherical curvature at $P$ of $V_{n}$ is equal to: (1) the product of the extreme mean curvatures of vector-fields in $V_{n}$ at $P$, (2) the square of the product of the extreme mean curvatures of asymptotic vector-fields in $V_{n}$ at $P$, (3) the product of the mean curvatures of the $n$ vector-fields at $P$ corresponding to the principal directions for the Ricci tensor $R_{i j}$ when $V_{n}$ is minimal. New interpretations of the Gaussian curvature of a surface $S$ in an ordinary space are obtained: (1) the Gaussian curvature of $S$ at $P$ is the product of the principal curvatures of any two distinct conjugate vector-fields in $S$ at $P,(2)$ the Gaussian curvature of $S$ at $P$ is the negative of the square of the magnitude of the Gaussian representation of a unit arc along an asymptotic line from $P$ in $S$. A simple proof of the theorem of Enneper is derived. (Received March 21, 1952.)

\section{LOGIC AND Foundations}

500. D. W. Bennett: $A$ set of axioms for the natural numbers.

Let $N$ be a set for which the following axioms are satisfied: I. There exists a mapping $f$ of the set of all nonempty subsets of $N$ onto $N$. II. For any nonempty sub- 
set $P$ in $N, f(P) \in P$. III. There exists a reversible mapping $g$ of the set $N$ onto the set of all elements of $N$ except $f(N)$. IV. For any subset $P$ of $N$ such that $f(P) \neq f(N)$, $g^{-1} f(P) \notin P$. The axioms are seen to involve no concepts except the familiar idea of mapping one set onto another. They lead immediately after appropriate definitions of " 1 " and "successor" to Peano's postulates for the natural numbers, and conversely. (Received March 12, 1952.)

501. E. W. Beth: Existence of complete models for extensions of the first-order predicate calculus.

Let $T$ be a complete extension of the first-order predicate calculus. Every set $S$ of expressions $A(x)$ containing a free variable $x$ defines, for every model $M$ of $T$, a subset $M(S)$ of the set of all elements of $M$. Let $S$ be finite; then, obviously, $M(S)$ will be nonempty if and only if $S$ is consistent with $T$. For an infinite set $S$ which is consistent with $T$, the set $M(S)$ will not, in general, be nonempty for every model $M$ of $T$. But it follows from the theorem of Löwenheim-Skolem-Gödel-Malcev that there is a model $M_{0}$ of $T$ which is complete in the following sense: for any set $S, M_{0}(S)$ will be nonempty if and only if $S$ is consistent with $T$. It follows that $M_{0}(S)$ is nonempty if and only if, for some model $M$ of $T, M(S)$ is nonempty. For $k=2,3, \cdots$ an analogous result can be proved for sets of expressions $A\left(x_{1}, x_{2}, \cdots, x_{k}\right)$ containing $k$ free variables. (Received March 12, 1952.)

\section{Statistics and Probability}

502. G. E. Forsythe and J. W. Tukey: The extent of $n$ random unit vectors.

Let $W_{1}, \cdots, W_{n}$ be random unit vectors which are independently and uniformly (with respect to $n-1$ dimensional measure) distributed on the unit sphere in $n$-dimensional space. Let $\Delta_{n}$ be the extent of these vectors, i.e., the signed $n$-dimensional volume of the parallelotope built on them. If $W_{i}$ has cartesian coordinates $w_{i j}$, then $\Delta_{n}$ $=\operatorname{det}\left(w_{i j}\right)$. A short geometrical proof of the following is given: for $n \geqq 2$ the ratio $\left|\Delta_{n} / \Delta_{n-1}\right|$ is the product of $n-1$ independent positive quantities, each of which has the same incomplete beta distribution $d F(u)=\Gamma(n / 2)\{\Gamma(n / 2-1 / 2) \Gamma(1 / 2)\}^{-1}$ $\cdot u^{(n-3) / 2}(1-u)^{-1 / 2} d u$; the ratios $\Delta_{n} / \Delta_{n-1}, \cdots, \Delta_{2} / \Delta_{1}, \Delta_{1}$ are independent; the odd moments $E\left(\Delta_{n}^{2 k+1}\right)=0$; the even moments $E\left(\Delta_{n}^{2 k}\right)=n !(n+2) ! \cdots(n+2 k-2) !\{0 ! 2 ! \cdots$ $(2 k-2) !\}^{-1}\{n(n+2) \cdots(n+2 k-2)\}^{-n}$. It is shown that the moments can also be obtained from the moments for the generalized variance; see S. S. Wilks, Biometrika vol. 24 (1932) pp. 471-494, esp. pp. 476-477. The second moment $E\left(\Delta_{n}^{2}\right)$ had been given by S. Krochmal [Leningrad. Universitet. Uchenye Zapiski vol. 83, Ser. Mat. Nauk 12 (1941) pp. 150-198; Mathematical Reviews vol. 7 (1946) p. 487]. (Received March 10, 1952).

\section{TOPOLOGY}

\section{3t. Barrett O'Neill: Essential sets and fixed points.}

Let $f$ be a self-map of a compact polyhedron $X$. A subset $A$ of $X$ is defined to be essential if every map in some neighborhood of $f$ in $X^{x}$ has a fixed point in $A$. Criteria are established that a subset be essential and that a subset contain an essential fixed point, as defined by M. K. Fort. The main tool is a fixed point index for sets similar to that of Leray and others. An elementary proof is given of the existence of an index and further properties derived. In special cases the essentiality of $A$ is related to the 
homological properties of $f, A$, and $X$. Maps of locally compact spaces are considered through their compactifications. Using Cech theory, the methods apply to nonpolyhedral spaces. (Received April 24, 1952.)

504t. R. H. Sorgenfrey: Mappings of plane convex sets.

It is an almost immediate consequence of a theorem of Borsuk that if $C$ is a compact convex set of width $w$ in a euclidian space and $f$ is a continuous real-valued mapping defined on $C$, then some inverse set under $f$ has diameter at least as great as $w$. It is shown that if $C$ is planar, then some inverse set has a component of diameter at least as great as $w\left(3^{1 / 2}\right) / 3$ in general and, in case $C$ is centrally symmetric, at least as great as $w\left(3^{1 / 2}\right) / 2$. Simple examples exist to show that these numbers cannot be improved. (Received March 12, 1952.)

W. T. Pucketr, Acting Associate Secretary 